López Núñez, J.A., Campos Soto, M.N., Aznar Díaz, I. \& Rodríguez Jiménez, C. (2020). Competencia digital del profesorado para la atención al alumnado con dificultades de aprendizaje. Una revisión teórica. Revista Electrónica Interuniversitaria de Formación del Profesorado, 23(2), 143-154.

DOI: https://doi.org/10.6018/reifop.418171

\title{
Competencia digital del profesorado para la atención al alumnado con dificultades de aprendizaje. Una revisión teórica
}

Juan Antonio López Núñez, María Natalia Campos Soto, Inmaculada Aznar Díaz, Carmen Rodríguez Jiménez

Universidad de Granada

\section{Resumen}

El uso de nuevas metodologías en el ámbito educativo, como es el uso de las tecnologías, influye positivamente en la reducción de las dificultades de aprendizaje que presenta el alumnado de Educación Primaria en general y en los que padecen algún tipo de trastorno como TDAH, en particular. De aquí nace el interés por la realización de este estudio, a través del cual se quiere analizar la situación actual de la literatura científica presente en la base de datos Scopus, en relación al uso de las TIC en educación con respecto a las dificultades de aprendizaje. Los resultados muestran que en los últimos cuarenta y cuatro años (1975-2019) las publicaciones existentes sobre el tema en cuestión han aumentado de forma significativa, pasando de publicar un documento en 1975 a producir cincuenta y nueve en 2019, es decir, se ha pasado de una producción de $0.014 \%$ a $8.79 \%$. La literatura científica muestra un primer repunte en el año 2010 (44 publicaciones) hasta llegar en 2019 a 59, lo que nos hace presagiar que seguirá aumentando en los próximos años. Finaliza la investigación proponiendo realizar otros estudios de características similares en las bases de datos Dialnet, Web of Science o Google Scholar.

Palabras clave: dificultades de aprendizaje; tecnología; competencia digital; TDAH.

\section{Contacto:}

María Natalia Campos Soto, ncampos@ugr.es, Facultad de Ciencias de la Educación Campus Universitario de Cartuja 18071 Granada, Este estudio forma parte de un proyecto de investigación financiado con fondos públicos del Ministerio de Educación, Cultura y Deportes del Gobierno de España, que tiene como título: «Implementación y mejora de procesos lectoescritores en alumnos con trastornos por déficit de atención e hiperactividad a través de las tecnologías de la información y la comunicación basadas en la animación 3D» (Referencia: FPU 16/06287). 


\title{
Digital competence of the teaching staff to attend to students with learning difficulties. A theoretical review
}

\begin{abstract}
The use of new methodologies in the educational field, such as the use of technology, has a positive influence on the reduction of learning difficulties presented by primary school students in general and those suffering from some type of disorder such as ADHD in particular. This is the origin of the interest in carrying out this study, through which we wish to analyse the current situation of the scientific literature present in the Scopus database, in relation to the use of ICT in education with respect to learning difficulties. The results show that in the last forty-four years (1975-2019) the existing publications on the subject in question have increased significantly, going from publishing one document in 1975 to producing fifty-nine in 2019 , that is, from a production of $0.014 \%$ to $8.79 \%$. The scientific literature shows a first increase in 2010 (44 publications) until reaching 59 in 2019, which makes us think that it will continue to increase in the coming years. The study ends by proposing to carry out a study of similar characteristics in the databases of Dialnet, Web of Science or Google Scholar.
\end{abstract}

Key words: learning disabilities; technology; digital competence; ADHD.

\section{Introducción}

Las dificultades de aprendizaje son consideradas necesidades específicas de apoyo educativo (NEAE), tal y como podemos comprobar en la Ley Orgánica 8/2013 (LOMCE) y en la Ley 17/2007 de Educación de Andalucía (LEA), ya que los sujetos afectados necesitan una atención educativa distinta a la ordinaria (González-Castellano, 2017). Este tipo de atención educativa hace referencia a aquel alumnado que:

Requiere, por un periodo de escolarización o a lo largo de toda ella, una atención educativa diferente a la ordinaria por presentar desórdenes significativos en los procesos cognitivos básicos implicados en los procesos de aprendizaje, que interfieren significativamente en el rendimiento escolar y en las actividades de la vida cotidiana del alumno o alumna y que no vienen determinados por una discapacidad intelectual, sensorial o motórica, por un trastorno emocional grave, ni por falta de oportunidades para el aprendizaje o por factores socioculturales. Por tanto, pueden presentarse simultáneamente pero no son el resultado de estas condiciones (Instrucciones del 8 de marzo de 2017, p. 160).

Dentro de este colectivo con dificultades de aprendizaje se encuentra el alumnado con trastorno por déficit de atención con hiperactividad (TDAH) el cual viene recogido en la LOMCE (2013) dentro del grupo con NEAE. Ahora bien, a raíz de las necesidades educativas que presente puede estar dentro de este colectivo, si precisa una atención educativa diferente a la ordinaria, o del alumnado con necesidades educativas especiales (NEE) en el caso de que sí necesite medidas específicas como es una adaptación curricular significativa (Instrucciones del 8 de marzo de 2017). 
No obstante, cabe destacar el uso de las tecnologías en la reducción de estas dificultades con el objetivo de mejorar los procesos de aprendizaje en estos sujetos. No debemos olvidar que vivimos en la sociedad digital por lo que los procesos de enseñanza deben ajustarse a los cambios constantes que estamos experimentando actualmente. Asimismo, los niños y jóvenes actuales son conocidos por Prensky (2001) como nativos digitales ya que han nacido en la era digital, por lo que el sistema educativo debe adaptarse a sus necesidades pero también a sus intereses. Numerosos autores apuestan por el uso de la tecnología como recurso didáctico para reducir las barreras en el aprendizaje que conducen a un alto porcentaje de estudiantes al fracaso escolar (García-Perales, Palomares-Ruiz y García-Perales, 2017; Estévez y León, 2014; Trigueros, Sánchez y Vera, 2012).

\section{Dificultades de aprendizaje en alumnado diagnosticado con TDAH de Educación Primaria}

Según Estévez y León (2015) "el TDAH se asocia a una serie de características que en el contexto escolar se convierte en necesidades específicas de apoyo educativo" (p. 230). Los síntomas nucleares del TDAH (desatención, hiperactividad e impulsividad) presentan un problema en el ámbito académico que es padecido tanto por las personas afectadas que ven cómo obtienen a diario peores resultados que sus compañeros y por sus familias que presencian cómo a pesar de los esfuerzos que realizan sus hijos, casi nunca alcanzan los objetivos marcados por el centro educativo. Estas dificultades que presentan nos hacen incluir a esta parte de la población dentro del colectivo en riesgo de fracaso escolar (INTEF, 2012). En general, los problemas académicos que presenta el alumnado con TDAH se relacionan principalmente con dificultades en la lectura (33\%) y dificultades en la escritura (63\%) con respecto a los que no padecen este trastorno (César-Mejía y Vilma -Varela2, 2015).

\section{Dificultades de aprendizaje relacionadas con la lectura}

Según la fundación CADAH (2012) aproximadamente un 20\% de los niños con TDAH presentan una comprensión lectora deficiente, en especial, si los textos son largos. Entre los errores que suelen cometer se encuentran: omisiones, sustituciones, adiciones, problemas con las sílabas trabadas, lectura a ritmo lento y con existencia de silabeo y rectificaciones.

\section{Dificultades de aprendizaje relacionadas con la escritura}

Además de presentar dificultades de aprendizaje con la lectura, por su sintomatología, también presentan dificultades con las instrucciones escritas y que, según la Fundación CADAH (2012), son, entre otras: divisiones incorrectas, omisiones, adiciones, sustituciones, caligrafía pobre, faltas ortográficas; desorganización, sustitución de letras, sílabas y palabras, los renglones ascienden o descienden.

\section{Dificultades de aprendizaje y tecnología}

Los seres humanos presentamos características comunes pero, también, rasgos que nos hacen diferentes, estas circunstancias están presentes en las personas de todas las culturas, lo que hace imprescindible la atención a la diversidad (Sampedro y McMullin, 2015). Precisamente, las TIC al adaptarse a las necesidades de cada persona responden a esta diversidad, influyendo en el desarrollo de la escuela inclusiva (Campos-Soto, López-Núñez y Marín, 2017). Nuestro sistema educativo valora de forma positiva la presencia de la diversidad en las aulas (García, 2016), estimando favorable el uso de las TIC para la reducción de las dificultades de aprendizaje que puedan presentar (García-Perales, Palomares-Ruiz y García-Perales, 2017; Rojas, Gómez y García, 2013; Luna, 2013) a la vez que 
confirman que el docente debe conocer las características de sus alumnos y aplicar las alternativas pedagógicas que considere más apropiadas para dar respuesta a sus necesidades (Cobas, Díaz y Navarro, 2014, citado en García-Pimentel, Puñales-Ávila y MuñozMartínez, 2019; Puñales-Ávila y Fundora-Martínez, 2017).

En años recientes son muchas las publicaciones que analizan el número, procedencia y otras características de investigaciones referentes a la tecnología de manera general (Van der Have y Rubalcaba, 2016), esto se debe no solo al auge de la temática en sí, sino a ser una línea de investigación que está adquiriendo cada vez más importancia en el ámbito investigador. Así, se pueden encontrar diferentes estudios que abordan temas relacionados como las TIC y la Realidad Aumentada (Arici, Yildirim, Caliklar \& Yilmaz, 2019; Martin, et al., 2011), las TIC y el TDAH (Chousa-Cortés, Martínez-Figueira y Raposo-Rivas, 2017) las dificultades del aprendizaje en sí mismas (Figueredo, Mazer, Guillaumon Emmel, Alba, 2014; Ram, 2018) y relacionadas con otras disciplinas educativas entre las que se incluye la tecnología (Fernández-Herrero, Lorenzo-Lledó, Carreres, 2018).

Teniendo en cuenta todo lo anterior, es necesario ver la realización de este estudio como una herramienta que tiene como objetivo el análisis de la influencia que tiene un elemento novedoso y cada vez más relevante, como es la tecnología dentro de la educación, en un área de estudio muy concreta como las dificultades del aprendizaje, todo ello a través del análisis de la literatura científica.

Fue a principios de 1990 cuando los investigadores comenzaron a tomarse interés por realizar este tipo de estudios (Miranda, 1990) a través de los cuales se podía obtener información sobre el curso de desarrollo de un área de conocimiento, productividad de las universidades y evolución de las revistas en un mismo ámbito (Rodríguez y Gallego, 2019).

\section{Metodología}

El método de investigación seguido en este estudio se basa en pautas propias marcadas por otras anteriores de carácter bibliométrico (Cruz, 1999; Fernández-Cano y Bueno, 1999; Pazos, Raposo-Rivas y Martínez-Figueira, 2015; Moreno-Guerrero, 2019).

La literatura fue consultada en la base de datos Scopus durante el primer trimestre del año 2020. La elección de esta base de datos se debe al rigor e importancia de su literatura científica, así como los diferentes mecanismos que establece para limitar y refinar los resultados de búsqueda según diversos indicadores.

Los metadatos obtenidos han sido obtenidos tras un análisis de otros estudios de la misma índole (Aznar, Trujillo y Romero, 2018; Braojos, Romera, Pérez Satorres y Cano, 2017; Gómez-García, Rodríguez-Jiménez y Ramos-Navas-Parejo, 2019):

- Año de publicación. Desde el origen hasta 2019. No se contempla el año 2020 al ser un año no finalizado.

- Autores más prolíficos.

- Instituciones más prolíficas.

- Tipos de publicación.

- Conexión de descriptores.

Para la elaboración de la muestra final $(n=671)$, se han llevado a cabo una serie de procesos. En un primer momento, los descriptores fueron delimitados por el tesauro de ERIC; en este caso los conceptos fueron "Learning disabilities" y "technology". Ambos 
descriptores fueron introducidos como ecuación de búsqueda con el operador booleano “AND" en la base de datos, con la finalidad de encontrar documentos de diversa índole que bien en su título, resumen o palabras clave contengan ambos descriptores.

\section{Resultados}

La búsqueda determinada en la base de datos anteriormente indicada da lugar a una combinación de documentos científicos de naturaleza variada, que oscilan desde el año 1975, primer año en el que aparecen documentos con dicha combinación de temáticas, hasta el año 2019.

La productividad diacrónica (figura 1) a lo largo de este tramo de tiempo muestra cómo la progresiva aparición de la tecnología en el ámbito de la educación en general, y de las dificultades del aprendizaje en particular, aumenta de manera significativa, siendo el número de documentos al inicio en 1975 de 1 (0.014\%) hasta llegar al máximo en 2019 con 59 documentos (8.79\%). Este aumento de la literatura se da sobre todo desde el año 2010, donde la producción de literatura científica a este respecto se duplica, verificándose así la premisa sobre la duplicidad de la literatura científica en periodos de 10-15 años (Price, 1986).

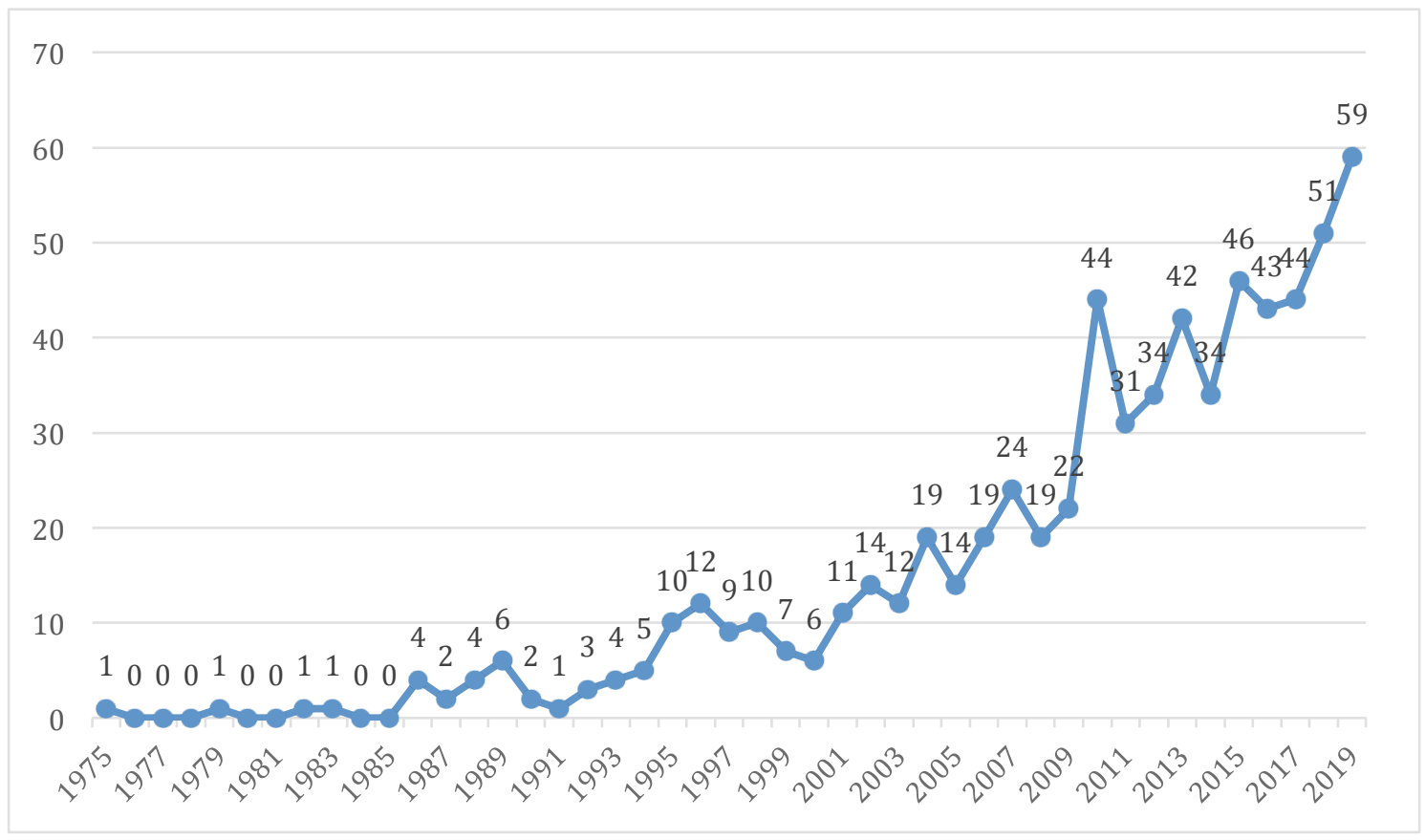

Figura 1. Productividad diacrónica sobre Tecnología y Dificultades del aprendizaje desde 1975 hasta 2019. Fuente: elaboración propia

\section{Indicadores de dispersión}

Si se atiende a los tipos de documentos a los que pertenece la totalidad de la literatura científica (figura 2), se observa cómo más de la mitad de estos son artículos, en concreto un total de 369 (55\%), dejando así a las comunicaciones en congresos en segundo lugar con 161 documentos (24\%) y siendo el resto de tipologías de documentos (reviews, capítulos de libro, libro... ) iguales o inferiores al 10\%. 


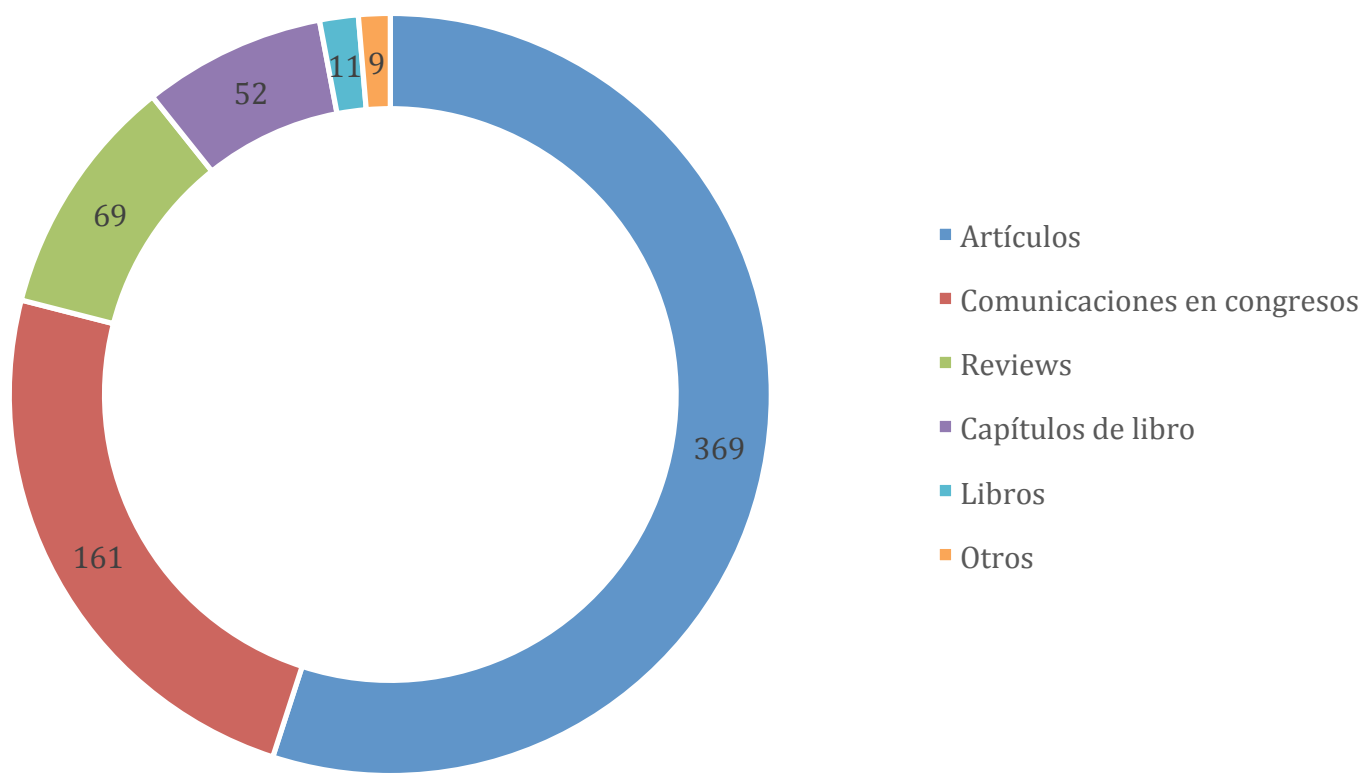

Figura 2. Clasificación de los documentos científicos según su tipología. Fuente: elaboración propia

\section{Indicadores de impacto}

Los indicadores de impacto muestran tres aspectos fundamentales de la literatura científica con el objetivo de dar a conocer la influencia o el papel que juega la tecnología en las dificultades del aprendizaje dentro del contexto educativo. Así, a continuación, se muestran los autores e instituciones más prolíficas, del mismo modo que un mapa de conexión de descriptores.

De este modo, se presentan los autores más prolíficos (tabla 1) en esta temática, esto es, aquellos con mayor número de publicaciones que versan sobre esta temática. En primer lugar, se encuentra Raskind M.H. con un total de 10 publicaciones (1.49\%), le sigue Evmenova A.S. con 9 publicaciones (1.34\%). Sin embargo, son otros autores con menor número de publicaciones los que destacan por obtener un mayor índice de impacto, en este caso es Williams P. el autor con un mayor índice, 298.16, derivado de 1789 citas de sus 6 publicaciones a este respecto.

Tabla 1.

Autores más prolíficos

\begin{tabular}{llllll}
\hline Autor & $\mathbf{N}^{\circ}$ doc & $\%$ & Citas & $\begin{array}{l}\text { Índice de } \\
\text { impacto }\end{array}$ & Universidad \\
\hline Raskind, M.H. & 10 & 1.490 & 477 & 47.7 & $\begin{array}{l}\text { Educational Consulting, } \\
\text { Bainbridge Island }\end{array}$ \\
Evmenova, A.S. & 9 & 1.341 & 137 & 15.2 & $\begin{array}{l}\text { Universidad George Mason, } \\
\text { Virginia, Estados Unidos }\end{array}$ \\
Poobrasert, O. & 8 & 1.192 & 18 & 2.25 & $\begin{array}{l}\text { Agencia nacional tailandesa de } \\
\text { Desarrollo Científico y }\end{array}$ \\
\hline
\end{tabular}




\begin{tabular}{|c|c|c|c|c|c|}
\hline & & & & & Tecnológico, Tailandia \\
\hline Bouck, E.C. & 7 & 1.043 & 977 & 122.12 & Universidad estatal de Michigan \\
\hline Bryant, B.R. & 7 & 1.043 & 796 & 113.71 & $\begin{array}{l}\text { The University of Texas at } \\
\text { Austin, Estados Unidos }\end{array}$ \\
\hline Higgins, E.L & 7 & 1.043 & 431 & 61.57 & Frostig Center, Estados Unidos \\
\hline Satsangi, R. & 6 & .894 & 193 & 32.16 & $\begin{array}{l}\text { George Mason University, } \\
\text { Virginia, Estados Unidos }\end{array}$ \\
\hline Wiliams, P. & 6 & .894 & 1789 & 298.16 & University College de Londres \\
\hline Aspinall, A. & 5 & .745 & 54 & 10.8 & Home Farm Trust, Reino Unido \\
\hline $\begin{array}{l}\text { Benmarrakchi, } \\
\text { F. }\end{array}$ & 5 & .745 & 24 & 4.8 & $\begin{array}{l}\text { Universidad Chouaib Doukkali, } \\
\text { Marruecos }\end{array}$ \\
\hline
\end{tabular}

Si se atiende a las instituciones que destacan en términos de producción científica (tabla 2), se comprueba cómo la universidad George Mason y la universidad de Texas, ambas de Estados Unidos, las que destacan notablemente con respecto al resto de instituciones en número de publicaciones. Por otro lado, si se atiende al índice de impacto o número de citas son otras instituciones las que destacan, en este caso es la universidad de Kansas y la de Toronto las que obtienen los valores más altos a este respecto.

Tabla 2.

Instituciones más prolíficas

\begin{tabular}{|c|c|c|c|c|}
\hline Instituciones & $\mathrm{N}^{\circ}$ doc & $\%$ & Citas & Índice de impacto \\
\hline $\begin{array}{l}\text { Universidad George Mason, } \\
\text { Estados Unidos }\end{array}$ & 17 & 2.533 & 477 & 47.7 \\
\hline $\begin{array}{l}\text { Universidad de Texas, } \\
\text { Austin, Estados Unidos }\end{array}$ & 16 & 2.384 & 137 & 15.2 \\
\hline Frostig Center, California & 10 & 1.490 & 18 & 2.25 \\
\hline Universidad de Oregón & 9 & 1.341 & 977 & 122.12 \\
\hline Universidad de Toronto & 9 & 1.341 & 796 & 113.71 \\
\hline $\begin{array}{l}\text { University College de } \\
\text { Londres }\end{array}$ & 9 & 1.341 & 431 & 61.57 \\
\hline Universidad de Delaware & 8 & 1.192 & 193 & 32.16 \\
\hline Universidad de Kansas & 7 & 1.043 & 1789 & 298.16 \\
\hline Universidad de Maryland & 7 & 1.043 & 54 & 10.8 \\
\hline $\begin{array}{l}\text { Universidad de Temple, } \\
\text { Pensilvania }\end{array}$ & 7 & 1.043 & 24 & 4.8 \\
\hline
\end{tabular}

Finalmente, la figura 3 muestra las conexiones entre los diferentes descriptores de todos los documentos sobre la temática que se trata. El mapa bibliométrico expuesto está compuesto por redes de nodos que muestran las palabras clave de los documentos que componen la muestra total. Para la realización de dicho mapa se ha utilizado el software VOSviewer. Aquellos nodos que de mayor tamaño y con las uniones más grandes reflejan que esos descriptores y esas conexiones han sido utilizadas en más ocasiones que aquellos que aparecen en menos tamaño. Los colores diferencian tipos de clústeres que se interrelacionan con otros, dando lugar entonces a zonas de colores intermedios. 
Así, se puede apreciar cómo son dos grupos los principales, en este caso los de color rojo y verde, siendo las palabras clave "learning disabilities" y "human" las que se destacan de cada uno de ellos respectivamente. En un segundo plano, está el grupo de color azul donde la palabra clave principal es "learning disorders", seguida de otras muchas relacionadas con la educación.

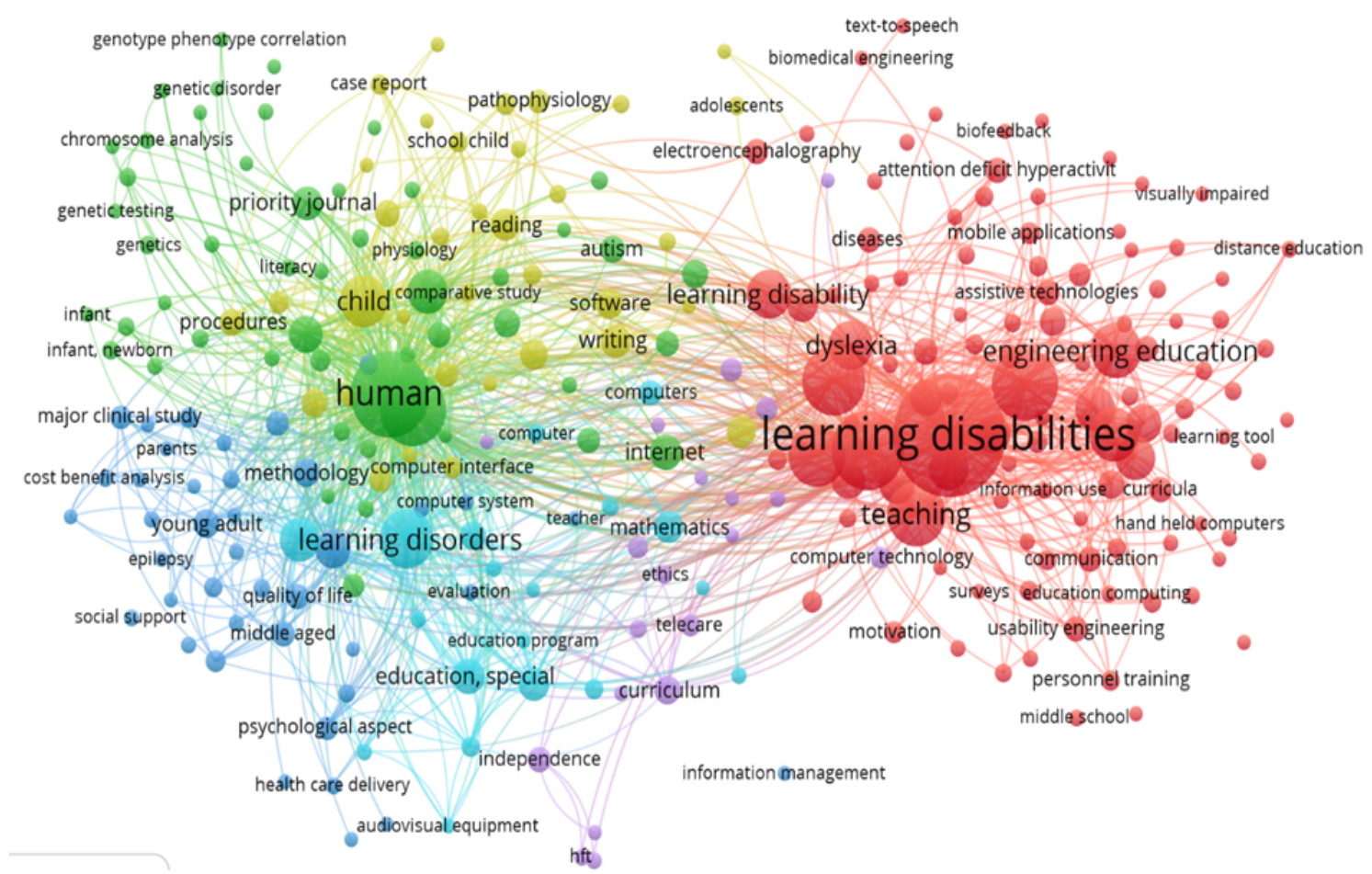

Figura 3. Mapa de redes entre descriptores sobre Tecnología y Dificultades de Aprendizaje

\section{Discusión y conclusiones}

Haciendo referencia a las dificultades de aprendizaje que presentan ciertos alumnos, entre los que se encuentran los diagnosticados con TDAH, las tecnologías han tenido gran transcendencia en la última década ya que numerosos estudios han demostrado cómo influyen en la mejora del proceso de enseñanza y aprendizaje (García-Perales, PalomaresRuiz y García-Perales, 2017; Estévez y León, 2014).

Atendiendo al tema en cuestión, los resultados de la productividad diacrónica en los últimos 44 años muestran que entre el año 1975 y el 2019 se produjo una progresiva aparición de la tecnología como recurso didáctico para paliar las dificultades de aprendizaje, presentando un aumento significativo, pasando de publicar 1 documento a principios de 1975 (0.014\%) a producir 59 en 2019 (8.79\%), lo que demuestra que se cumple la Ley de Price que sostiene que la producción científica se duplica cada 10-15 años. Como se puede comprobar en la figura 1, en 1975 se publicó 1 documento, en 1989 se publicaron 6, pasando a difundirse 19 en 2003 y ascendiendo la producción en 2019 a 59. Estos resultados coinciden con (GarcíaPerales, Palomares-Ruiz y García-Perales, 2017) que afirman que cada vez se está más convencido de los resultados positivos del uso de las TIC en la reducción de las dificultades de aprendizaje. 
En lo que concierne a la tipología de los documentos científicos, se observa que la mayoría de las publicaciones son artículos (55\%) seguido de comunicaciones en congresos (24\%), en tercer lugar se encuentran las revistas (10\%), a bastante distancia los capítulos de libro (8\%), encontrándose en un porcentaje alejado los libros (2\%) y otros documentos (1\%), lo que demuestra que los investigadores apuestan por los artículos científicos con respecto al tema en cuestión.

Con respecto a los autores con mayor producción científica sobre esta temática encontramos que los que tienen mayor número de publicaciones son Raskind M.H. (1.49\%) y Evmenova A.S. (1.34\%). Sin embargo, los autores que destacan, de manera considerable, con mayor índice de impacto son Williams P. con un índice de 298.16, derivado de 1789 citas de sus 6 publicaciones y Bouck, E.C. con un índice de 122.12 a raíz de 977 citas de sus 7 producciones, lo que determina que estas últimas publicaciones constituyen el núcleo de la disciplina ya que acumulan el $50 \%$ de las citas (Ardanuy, 2012).

Atendiendo a las instituciones que destacan en términos de producción científica se comprueba cómo sobresalen las dos universidades de Estados Unidos (Universidad George Mason y Universidad de Texas) con 17 y 16 publicaciones respectivamente. Sin embargo, y de forma similar a lo que ocurre con los autores más prolíficos, estas universidades no son las que tienen mayor índice de impacto ya que como se puede comprobar en la tabla 2 el mayor índice lo tienen la Universidad de Kansas (298.16) con 7 publicaciones y la Universidad de Oregón (122.12) con 9 publicaciones, seguido muy de cerca por la Universidad de Toronto (113.71) con 9 también.

Finalmente, y atendiendo a las conexiones de los distintos descriptores, podemos comprobar que los más utilizados han sido "learning disabilities" y "human", seguidos de "learning disorders", y en menor proporción encontramos "child", "education special","currículum", "assistive technologies", entre otras.

En conclusión, y de acuerdo con los datos obtenidos, cabe destacar que el uso de nuevas metodologías, como es el uso de las tecnologías en el ámbito educativo, influye positivamente en la reducción de las dificultades de aprendizaje que presenta el alumnado de Educación Primaria en general y en los que padecen algún tipo de trastorno como el TDAH, en particular. Como se ha podido comprobar, la literatura científica, en lo referente al uso de la tecnología para disminuir las dificultades de aprendizaje en el alumnado, presente en la base de datos Scopus muestra un primer repunte en el año 2010 (44 publicaciones) y otro aún mayor en 2019 (59) lo que nos hace presagiar que seguirá aumentando en los próximos años, por lo que, sería conveniente seguir profundizando en el tema en cuestión y seguir comprobando hasta qué punto es posible minimizar y, a ser posible, eliminar las dificultades de aprendizaje que presentan los alumnos de Educación Primaria y, más concretamente, los diagnosticados con TDAH, gracias al uso de las tecnologías, alcanzando la finalidad del sistema educativo que es el desarrollo integral de nuestros discentes.

El uso de las TIC como recurso didáctico puede ser favorable para la inclusión de alumnado con NEAE evitando la exclusión y fomentando la participación de todos los estudiantes, independiente de sus características personales (Estévez y León, 2014; Rojas, Gómez y García, 2013). De esta manera el beneficio no estaría orientado solamente a un determinado colectivo sino a todos los compañeros favoreciendo un clima de equidad (Luna, 2013; Trigueros, Sánchez y Vera, 2012).

Las limitaciones encontradas en el desarrollo de la investigación se centran en que no se encontraron documentos que relacionaran: "dificultades de aprendizaje" "tecnologías" y 
"TDAH" en la base de datos Scopus por lo que la búsqueda se limitó a los dos primeros descriptores que eran los que más documentos presentaban registrados en Scopus.

Como futura línea de investigación sería aconsejable llevar a cabo un estudio de características similares en las bases de datos de Dialnet, Web of Science o en Google Académico.

\section{Referencias}

Ardanuy Y. (2012). Breve introducción a la bibliometría. Barcelona, España: Universitat de Barcelona.

Arici, F., Yildirim, P., Caliklar, Ş., y Yilmaz, R. M. (2019). Research trends in the use of augmented reality in science education: Content and bibliometric mapping analysis. Computers \& Education, 142, 103647.

Aznar Díaz, I., Trujillo Torres, J. M., \& Romero Rodríguez, J. M. (2018). Estudio bibliométrico sobre la realidad virtual aplicada a la neurorrehabilitación y su influencia en la literatura científica. Revista Cubana De Información En Ciencias De La Salud, 29(2), 1-10.

Braojos, C. G., Romera, A. M., Pérez, H. S., Satorres, A. V. C., \& Cano, A. F. (2017). Análisis temático de la investigación educativa soportada por Grounded Theory. Bordón. Revista de pedagogía, 69(1), 83-102.

Campos-Soto, M.N., López-Núñez, J.A., Marín, J. A. (2017). Funcionalidad de las TIC en el proceso de enseñanza y aprendizaje en niños diagnosticados con TDAH. En RuizPalmero, J., Sánchez-Rodríguez, J. y Sánchez-Rivas, E. (Edit.). Innovación docente y uso de las TIC en educación. Málaga, España: UMA Editorial.

César-Mejía Z., \& Vilma -Varela C. (2015). Comorbidity of reading and writing learning. Disabilities in children diagnosed with ADHD. Psicología desde el Caribe, 1, 121-144.

Chousa-Cortés, C., Martínez-Figueira, M.E. y Raposo-Rivas, M. (2017). Las TIC para la intervención educativa en TDAH: un estudio bibliométrico. Perspectiva Educacional. Formación de Profesores, 56(3), 142-161.

Cruz, M. (1999). Bibliometría y Ciencias Sociales. Clío: History and History Teaching, 7, 1-10.

Estévez, B. y León, M.J. (2015). La inclusión educativa del alumnado con TDA/TDAH. Rompiendo con las barreras curriculares y organizativas de los centros escolares de Educación Primaria. [tesis doctoral]. Granada, Universidad de Granada.

Estévez, B. y León, M.J. (2014). Una mirada inclusiva hacia el TDAH en las aulas escolares. XI Congreso Internacional y XXXI Jornadas de Universidades y Educación Inclusiva. Quaderns Digitals.net. Recuperado de http://www.quadernsdigitals.net/index.php?accionMenu=hemeroteca.DescargaArtic ulolU.descarga\&tipo=PDF\&articulo_id $=11346 \&$ PHPSESSID $=06$ a $5516 \mathrm{fbf} 575 \mathrm{bdedo} 997 \mathrm{fz}$ d71928016

Fernández-Cano, A., y Bueno, A. (1999). Synthesizing scientometric paterns in Spanish educational research. Scientometrics, 46(2), 349-367.

Fernández-Herrero, J., Lorenzo-Lledó, G., Carreres, A. L. (2018). A bibliometric study on the use of virtual reality (VR) as an educational tool for high-functioning Autism Spectrum Disorder (ASD) children. Contemporary Perspective on Child Psychology and Education, 59-80. 
Figueredo, M.D.O., Mazer, S.M., Guillaumon Emmel, M.L., Alba, E.F. (2014). Scientific production on learning disabilties in Brazil: A literatura Review. Aula Abierta, 42(1), 3138. doi:10.1016/S0210-2773(14)70006-X

Fundación Cadah (2012). TDAH en la infancia: Detección precoz, diagnóstico y tratamiento. [blog]. Recuperado de https://www.fundacioncadah.org/web/articulo/tdah-en-lainfancia-deteccion-precoz-diagnostico-y-tratamiento.html

García-Perales, R., Palomares-Ruiz, A. y García-Perales, R. (2017). La respuesta individualizada de las dificultades de aprendizaje y las TIC. En Palomares-Ruiz. A. (coord.), Una mirada internacional sobre la educación inclusiva (pp. 26-33). Castilla La Mancha, España: Ediciones de la Universidad de Castilla-La Mancha, http://doi.org/10.18239/jor_08.2017.01

García-Pimentel, L., Puñales-Ávila, C.L. y Muñoz-Martínez, C.A. (2019). Del proceso formativo del maestro primario, una mirada desde la didáctica de la matemática. Matanzas, Cuba: Universidad de Matanzas. ISBN: 978-959-16-4317-9

García, R. (2016). Sexo femenino y capacidades matemáticas: desempeño de los más capaces en pruebas de rendimiento matemático. Ensaio: Avaliação e Políticas Públicas em Educação, 24 (90), 1-25.

Gómez-García, G., Rodríguez-Jiménez, C., \& Ramos-Navas-Parejo, M. (2019). Virtual Reality in Physical Education area. Journal of Sport and Health Research. 11(Supl 1), 177-186.

González Castellano, N. (2017). Inteligencias múltiples y dificultades de aprendizaje. MLSEducational Research, 1 (1), 19-38. DOI: 10.29314/mlser.v111.26

Instrucciones de 8 de marzo de 2017, de la dirección general de participación y equidad, por las que se actualiza el protocolo de detección, identificación del alumnado con necesidades específicas de apoyo educativo y organización de la respuesta educativa. Sevilla. Recuperado de https://www.juntadeandalucia.es/educacion/portals/abacoportlet/content/fa188460-6105-46b1-a5do-7eegb19526df

Ley Orgánica 8/2013, de 9 de diciembre, para la mejora de la calidad educativa. BOE 295, 10/12/2013.

Ley 17/2007, de 10 de diciembre, de Educación de Andalucía. BOJA 252, 26/12/2007.

Luna, M.R. (2013). Tecnología y discapacidad: Una mirada pedagógica. En RDU, Revista Digital Universitaria, $14 \quad$ (12), 1-19. Recuperado de http://www.revista.unam.mx/vol.14/num12/art53/

Martin, S., Diaz, G., Sancristobal, E., Gil, R., Castro, M., y Peire, J. (2011). New technology trends in education: Seven years of forecasts and convergence. Computers \& Education, 57(3), 1893-1906.

Ministerio de Educación, Cultura y Deporte. INTEF (2012). Respuesta educativa para alumnado con TDAH. Realidad educativa de los alumnos con TDAH. Recuperado de http://formacion.educalab.es/mod/page/view.php?id=20136

Miranda, A. (1990). Bibliometría. Bibliotecas, 8(1), 1-11. Recuperado de: https://revistas.una.ac.cr/index.php/bibliotecas/article/view/761

Moreno-Guerrero, A.J. (2019). Estudio bibliométrico de la Producción Científica sobre la Inspección Educativa. REICE. Revista Iberoamericana sobre Calidad, Eficacia y Cambios en Educación, 17, 23-40. https://doi.org/10.15366/reice2019.17.3.002 
Pazos, M., Raposo-Rivas, M. y Mar nez-Figueira, E. (2015). Las TIC en la educación de las personas con Síndrome de Down: un estudio bibliométrico. Virtualidad, Educación y Ciencia, 11(6), pp. 20-39. Recuperado de https://revistas.unc.edu.ar/index.php/vesc/article/view/12767

Prensky, M. (2001). Digital natives, digital immigrants. On the Horizon, 9(5), 1-6.

Price, D.J.S. (1986). Little Science, big science... and beyond. Nueva York, NY: Columbia University Press.

Puñales-Ávila, L. y Fundora-Martínez, C.L. (2017). La enseñanza de la lectoescritura en la Educación Primaria: reflexión desde las dificultades de aprendizaje. Atenas. Revista científico pedagógica, 1(37), 125-138.

Ram, S. (2018). "Word Blindness" (Dyslexia): A Bibliometric Analysis of Global Research in Last Fifty Years. DESIDOC Journal of Library \& Information Technology, 38(4), 286.

Rodríguez, A., y Gallego, J.L. (2019) Análisis Bibliométrico sobre Educación Especial. Profesorado. Revista de Currículum y Formación del Profesorado, 23(1), 307-327. doi:10.30827/profesorado.v23i1.52458

Rojas, G., Gómez, M.G. y García, N.J. (2013). El uso de un software educativo para promover el aprecio por la diversidad en alumnos de primaria. Revista Apertura, 5 (2), 16-29. Recuperado http://www.udgvirtual.udg.mx/apertura/index.php/apertura/article/view/406/331

Sampedro, B. y McMullin, K. (2015). Videojuegos para la inclusión educativa. Digital Education Review, (27), 122-137.

Trigueros, F.J., Sánchez, R. y Vera, M.I. (2012). El profesorado de Educación Primaria ante las TIC: realidad y retos. REIFOP. Revista electrónica interuniversitaria de formación delprofesorado, 15(1), 101-112.

Van der Have, R. P. y Rubalcaba, L. (2016). Social innovation research: An emerging area of innovation studies? Research Policy, 45(9), 1923-1935. 